\title{
OS MÉTODOS MISTOS E A INTERDISCIPLINARIDADE NAS CIÊNCIAS SOCIAIS: PRAGMATISMO OU PLURALISMO PARADIGMÁTICO?
}

Roberto Donato da Silva Júnior*

e Álvaro de Oliveira D'Antona **

\begin{abstract}
Resumo: $\mathrm{O}$ artigo testa se a interdisciplinaridade nas ciências sociais associa-se a métodos particulares em artigos científicos. Partindo da hipótese de afinidade entre interdisciplinaridade e métodos mistos, nós buscamos os 30 artigos em ciências sociais mais citados no ISI-WebofScience (1990/2013) com o termo 'mixed methods'. Nós não encontramos menções à palavra "interdisciplinarity" ou a outras similares em tais artigos, o que nos leva a analisar os motivos da não correspondência entre os termos.

Palavras-chave: ciências sociais; interdisciplinaridade; métodos mistos; pragmatismo; pluralismo.

Keywords: The paper tests whether interdisciplinarity is associated with particular methods in the social sciences. Assuming association between interdisciplinarity and mixed methods, we searched the 30 most cited social sciences' papers on ISIWebofScience (1990/2013) using the term 'mixed methods'. We found no references to the word "interdisciplinary" or to other similar words in such papers, which leads us to analyze the reasons for the mismatch between the terms.
\end{abstract}

Abstract: interdisciplinarity; mixed methods; pragmatism; pluralism; social sciences.

\footnotetext{
* Cientista Social, Doutor em Ambiente e Sociedade (NEPAM/UNICAMP) e Professor do Centro de Ciências Humanas e Sociais Aplicadas (CCHSA) da PUC-Campinas. E-mail roberto.junior@puc-campinas.edu.br

** Professor da Faculdade de Ciências Aplicadas (FCA-Unicamp) e do Programa de Pós-graduação de Demografia (IFCH-Unicamp) E-mail: alvaro. dantona@fca.unicamp
} 
$|88|$

Os métodos mistos e a interdisciplinaridade nas....

\section{Introdução}

Mesmo que o método não seja suficiente para definir uma disciplina, determinadas formas de coleta e tratamento de dados são imediatamente associáveis a disciplinas específicas das ciências sociais (por exemplo, a etnografia e a antropologia; a coleta censitária e a demografia). Assim, se há um possível encontro entre escopo disciplinar e especificidade metodológica, é presumível que estudos interdisciplinares estejam afinados a aplicação de métodos mistos (mixed methods). Um olhar mais atento à produção bibliográfica sobre mixed methods e interdisciplinaridade confirma a hipótese?

No artigo, nos perguntamos se a interdisciplinaridade nas ciências sociais - representadas aqui pela demografia, sociologia e antropologia - está relacionada à aplicação de métodos particulares de forma combinada. Partimos de duas grandes discussões não necessariamente tratadas conjuntamente pela bibliografia: i) a interdisciplinaridade como uma necessidade para dar conta de problemas complexos da modernidade tardia, como os apresentados pelas questões ambientais; ii) os métodos mistos como uma forma privilegiada de integração/complementação de formas 'típicas' de coleta e tratamento de dados.

Para a análise, consideramos artigos publicados em revistas indexadas no ISI-WebofScience entre 1990-2013 na área de ciências sociais. Inicialmente foram selecionados os trinta artigos mais citados nos quais o termo 'mixed methods' figura no título ou em tópicos. Nestes, procuramos aqueles que continham os termos "interdisciplinary", "interdisciplinarity", "multidisciplinarity" e "multidisciplinar". A intenção foi evidenciar o tipo de relação que esses artigos propunham para os termos. Não foi encontrado um único artigo que se refira explicitamente aos termos, indicando que não há uma relação automática entre experiências de mixed methods e práticas de interdisciplinaridade, apesar de reconhecidos esforços nesse sentido (Johnson e Onwuegbuzie, 2004). Como explicar essa lacuna? 
Assim, recorremos a duas inserções teóricas paralelas: primeiro, a investigação sobre os rumos da interdisciplinaridadenas ciências sociais - demografia, antropologia e sociologia - de modo a captar o nexo epistemológico que permita compreender certo alheamento de exercícios de interdisciplinaridade à combinação metodológica; segundo, a prospecção das formas potenciais de combinações de métodos que possibilitem a redefinição dos contornos da interdisciplinaridade comumente exercida.

O primeiro exercício, constituído de uma inserção mais geral sobre as ciências sociais e das análises precedentes (Silva Junior, 2012; Silva Junior e D'Antona, 2012; Silva Junior e Ferreira, 2013) circunscritas à questão da interdisciplinaridade na sociologia, antropologia e demografia sobre sustentabilidade demonstra que, de forma geral, as propostas de interdisciplinaridade se constituem por um processo aqui denominado de "interdisciplinaridade disciplinar", ou seja, diferentes perspectivas científicas tendem ao "diálogo" quando compartilham os mesmos pressupostos epistemológicos, possibilitando o não enfrentamento de estranhamentos discursivos, temáticos e teórico-metodológicos. Essa continuidade epistêmica operacionaliza um modelo de análise possível de interações sem abrir mão, contudo, de certa homogeneidade de apreensão empírica, formulação conceitual e destilação teórico-metodológica. Promove-se, assim, um processo interdisciplinar sem romper a dinâmica disciplinar que constituem as diferentes ciências.

O segundo exercício explora a capacidade dos métodos mistos em mediar formas prospectivas diferenciadas, seja de perspectivas quali-quanti, da relação entre análise documental e etnográfica ou entre diferentes noções de espacialidade (desenhos e mapas), no contexto da preocupação de um campo metodológico institucionalizado. Assim, evidencia-se a capacidade dos métodos mistos em cobrir lacunas analíticas normalmente existentes em empreendimentos metodológicos únicos.

Se, por um lado, encontramos uma "interdisciplinaridade disciplinar" e, por outro, métodos mistos orientados para os interstícios, um esforço de aproximação entre os dois temas podem 
$|90|$

Os métodos mistos e a interdisciplinaridade nas....

ser fundamental para redefinição das relações entre ciências, construindo-se, assim, os caminhos para uma interdisciplinaridade de "confronto e enfrentamento" entre perspectivas científicas que se mantêm tradicionalmente distantes. Sob essa orientação, talvez, o inevitável encontro entre ciências sociais e naturais no escopo das questões ambientais pode ser aprofundado sem a reprodução de perspectivas antagônicas, mas complementares, que temem ou advogam a redução de uma dimensão à outra.

\section{O ponto de partida}

Essa pesquisa busca discutir os possíveis debates sobre interdisciplinaridade realizados em artigos sobre métodos mistos. Através do sítio ISI - WebofScience, realizou-se uma pesquisa sobre os trinta artigos mais citados, por título e tópicos, entre 2009 e 2013, nas seguintes áreas: "anthropology", "social issues", "social sciences other topics", "sociology", "demography". Dos trinta artigos encontrados, foi realizado um segundo nível de pesquisa, no qual foram "buscados" os termos: "interdisciplinarity", interdisciplinary" e "multidisciplinarity" e "multidisciplinary".

O resultado dessa prospecção é que em nenhum dos artigos foram encontrados os termos da busca. Bem entendido, isso não significa que nos textos iniciativas e produtos interdisciplinares não estivessem contemplados. Mas, antes, que não existem nesses artigos a problematização da relação entre disciplinaridade e interdisciplinaridade. Qual seria a razão do não encontro entre a problemática interdisciplinar e o campo dos métodos mistos? Quais seriam os dilemas enfrentados pelas abordagens interdisciplinares e como uma aproximação as iniciativas de combinação metodológica poderiam contribuir para resolução dessas limitações? Por outro lado, se interdisciplinaridade não é uma questão, quais são problemas enfrentados por autores que se dedicam a refletir sobre métodos mistos como um campo institucionalizado, no que se refere às necessidades que leva à busca de procedimentos combinatórios? A aproximação ao debate 
sobre interdisciplinaridade contribuiria, de alguma forma, com essas questões?

No que se segue, o artigo busca abordar essas questões, de modo que suas possíveis respostas possam orientar pesquisas mais sistemáticas sobre a relação entre disciplinaridade e métodos combinados, tanto do ponto de vista socioepistemológico - ou seja, partir da relação entre sociologia da ciência e epistemologia quanto do ponto de vista da aplicação de métodos mistos com uma expressão de experiências efetivas sobre interdisciplinaridade. Nossa hipótese é de que essas possibilidades podem contribuir para enriquecer o escopo no qual as questões ambientais são atualmente pensadas.

\section{Interdisciplinaridades e ciências sociais}

Encontrar uma origem para o termo interdisciplinaridade é tarefa pouco frutífera, mas, seguramente, sua emergência só faz sentido nos quadros de formação cientificismo do século XIX, onde a estrutura científica disciplinar se institucionaliza. Se, sob a perspectiva contemporânea, o encontro entre disciplinaridades é frequentemente encarado como uma solução para as consequências desastrosas da hiperespecialização, talvez seja pertinente ressaltar o caráter formativo que a interseção interdisciplinar desempenhou para a própria emergência das especializações científicas. Vide a original articulação entre filosofia hegeliana, teoria socialista e economia clássica promovida por Marx; a adoção do conceito de afinidades eletivas, emprestado à química e à filosofia (Howe, 1978), empreendida por Weber para a formação da sociologia compreensiva; e a interdisciplinaridade autoevidente da "fisiologia social" durkheimiana (Durkheim, 1972). Do mesmo modo, pode-se observar o princípio da população de Malthus fundar a demografia a partir de uma derivação de pensamento econômico produzido pelo autor.

Assim, a contemporânea noção integrativa (transdisciplinaridade) ou colaborativa (interdisciplinaridade) 
|92|

Os métodos mistos e a interdisciplinaridade nas....

entre ciências (Klein, 1990) que se firma e se difunde no pósguerra, pode ser compreendida não como um efeito da saturação do aspecto tecnocientífico da modernidade, mas, antes, como um elemento constitutivo da ambivalência própria do pensamento científico. Teríamos assim, uma incessante dinâmica de interação entre disciplinaridade e interdisciplinaridade no bojo da configuração epistêmica científica. Desse modo, parece lícito conceber que de uma interdisciplinaridade formativa, que fecunda disciplinaridades, emerge uma interdisciplinaridade reflexiva, que busca enfrentar os dilemas provocados pelas consequências dos processos de especialização cientifica e sua limitada potencialidade em lidar com essas derivações.

O ponto de inflexão entre essas duas interdisciplinaridades encontra-se na década de 1940, tendo como "ritual de passagem" as "Conferências Macy", uma série de encontros entre reconhecidos intelectuais da época - envolvendo matemáticos, físicos, antropólogos, biólogos e outros - que tinham por objetivo fundar uma ciência da mente, naquele momento, denominada de cibernética. De forma geral, o fracasso (Dupuy, 1996; Heims, 1991) da iniciativa não impediu que novos sentidos de colaboração entre experts de diferentes matizes tomasse fôlego, pois no impulso de experimentar um exercício de interdisciplinaridade formativa, os cientistas ali reunidos tinham na sua frente um dilema propriamente motivador das formas de interdisciplinaridade reflevixa: o enfrentamento do complexo, do furtivo e do teimosamente indeterminável pelos processos de purificação científica. Como resultado, ao invés de uma única ciência da mente, das Conferências Macy surgiu uma configuração de disciplinas, denominadas, de forma geral de ciências cognitivas (Dupuy, 1996). As decorrências para as ciências sociais foram as mais diversas: a aventura interdisciplinar da ecologia da mente batesoniana (Bateson, 1972), o fundamento disciplinar do estruturalismo levistraussiano (Lévi-Strauss, 1985) e o projeto transdisciplinar da complexidade moriniana (Morin, 1973).

Assim, a interdisciplinaridade reflexiva, como trânsito entre temas e orientações teórico-metodológicas institucionalizadas 
em disciplinas, ganha impulso por ser considerada uma ferramenta eficaz para entendimento de problemas considerados complexos. Como compreender os híbridos socioambientais, as novas configurações sociotécnicas ou o descentramento das identidades? A profusão, não somente nas ciências sociais, de estudos autodeclarados interdisciplinares, não seguiu um método ou orientação teórica comum (Klein, 1990). No interior das ciências sociais, a antropologia tem se apresentado como protagonista das tentativas de superação não somente das fronteiras entre ciências, mas também entre os campos ontológicos edificados pela modernidade, em especial, as relações entre "natureza e sociedade". Essa tentativa se apresenta tanto entre as tendências ecológicoculturais (Moran, 1994) - no qual o conceito de adaptação tem papel central como elemento articulador -, quanto em perspectivas sociossimbólicas, tendo o conceito de rede (Latour, 1994), trama (Ingold, 2011) ou esquemas de práxis (Descola, 2001) como princípio norteador das tentativas de integração. A sociologia, por seu turno, não está indiferente às perspectivas de interdisciplinaridade. Beck (2010), por exemplo, propõe o abandono da hiperespecialização nas ciências pela especialização nos contextos, no qual as diferentes perspectivas científicas dialoguem pelo princípio da aprendizagem em torno dos problemas apresentados pela dinâmica amalgamada dos riscos contemporâneos.

Se a conceituação aqui proposta-de uma interdisciplinaridade formativa e outra reflexiva - é pertinente, cabe, então, indagar: a interdisciplinaridade reflexiva tem sido bem sucedida em sua pretensão em solucionar as restrições demonstrada pela disciplinaridade diante de problemas complexos?

Em pesquisas que buscam apreender o caráter híbrido e interdisciplinar das propostas contemporâneas de sustentabilidade que cobre ecologia, sociologia, antropologia e demografia (Silva Junior, 2012; Silva Junior e D'Antona, 2012; Silva Junior e Ferreira, 2013, Silva Junior, 2013), temos percebido um processo de polarização das demandas de interdisciplinaridade em dois grandes polos epistêmicos: ecológico e sociológico. 
|94|

Os métodos mistos e a interdisciplinaridade nas....

O primeiro traz para sua esfera, fundamentalmente, a economia e demografia. Isso significa que os processos de interdisciplinaridade entre essas disciplinas se estruturam a partir de programa teórico-conceitual e semântico definido pela ecologia Entre ecologia e economia, por exemplo, existe um caráter de continuidade, na medida em que operam no mesmo "registro" epistemológico. Suas categorias analíticas se relacionam sem maiores disparidades conceituais e sem grandes campos de conflituosidade discursiva. Esse caráter de continuidade se expressa nos conceitos de "estrutura" e "função" ecológica, relacionados respectivamente com os conceitos de "bens" e "serviços" econômicos. Do mesmo modo, a articulação entre "ecossistema" e "capital natural", assim como entre "paisagem" e "cenário", encontram-se amplas possibilidades de diálogo interdisciplinar (Silva Junior e Ferreira, 2013). No caso da demografia, evidenciouse a ausência de um esquema teórico-metodológico próprio, sendo suas propostas de sustentabilidade constituídas a partir de um campo de análise estruturado pela ecologia, a partir da hegemonia do conceito de "capacidade de suporte" como forma de tratamento das interações entre população e ambiente, assim como a persistência da perspectiva neomalthusiana centrada no volume populacional como risco socioambiental predominante (Silva Junior e D’Antona, 2012).

O segundo polo, sociológico, trata das relações entre sociologia e antropologia como campos de convergência do debate sobre sustentabilidade. Isso se refere principalmente à antropologia, que tende a incorporar contextos urbanos e tecnocientíficos dimensões tradicionalmente tratadas pela sociologia-dentro do seu espectro de abordagem. Essa incorporação impulsiona um diálogo entre teoria social e abordagens antropológica interessadas na problemática ontológica de interações entre "natureza" e "cultura" (Silva Junior, 2013). Quanto à perspectiva sociológica sobre sustentabilidade, ela se apresenta como a mais disciplinar dentre as aqui apresentadas. Isso se deve à situação de ela se encontra (ainda) em um período de maturação de arcabouço teórico próprio quanto às questões ambientais. No entanto, o uso do conceito de 
"rede" em artigos sociológicos sobre sustentabilidade demonstra uma aproximação silenciosa à perspectiva mais contemporânea da antropologia.

Esses elementos possibilitam compreender os processos interdisciplinares envolvidos em questões de sustentabilidade a partir de certa homogeneidade de apreensão empírica, formulação conceitual e destilação teórico-metodológica. Ou seja, emerge daí um processo interdisciplinar sem romper a dinâmica disciplinar que as constituem. A partir do compartilhamento de pressupostos epistemológicos, perspectivas científicas encontram amplas possibilidades de "diálogo" sem necessariamente mergulhar em processos de superação de estranhamentos discursivos, temáticos e teórico-metodológicos. É nesse sentido que os dois polos aqui problematizados parecem apontar para uma espécie de "interdisciplinaridade disciplinar", pois essas diferentes perspectivas científicas tendem ao "diálogo" quando compartilham os mesmos pressupostos epistemológicos, possibilitando, assim, o não enfrentamento de estranhamentos discursivos, temáticos e teórico-metodológicos.

A interdisciplinaridade disciplinar apresenta-se como a mediação entre o modelo formativo e reflexivo de diálogo entre diferentes ciências. Ela não se configura como formativa, pois não almeja processos de purificação de fenômenos, estando orientada para resguardar a caráter complexo dos eventos. Contudo, ela busca cumprir o "dever" de enfrentamento reflexivo dos dilemas da especialização sem romper totalmente as barreiras da disciplinaridade. Esse segundo aspecto é especialmente problemático, pois, ao limitar processos interdisciplinares ao diálogo entre "iguais", dimensões importantes de fenômenos complexos ficam em segundo plano em seus constructos de interpretação científica. Esse é o caso da relação entre ecologia e economia quando se debruçam sobre temas de sustentabilidade. A recorrente preocupação, entre elas, quanto aos temas sociais envolvidos nos dilemas ambientais não sem converte, no entanto, em interesse e aproximação aos temas sociológicos (Silva Junior e Ferreira, 2013). 
$|96|$

Os métodos mistos e a interdisciplinaridade nas....

Como, então, ultrapassar as barreiras apresentadas pela interdisciplinaridade disciplinar? De que modo poderíamos construir uma interdisciplinaridade de confronto e do estranhamento para, assim, dedicar-se às descontinuidades e lacunas de entendimento sobre a complexidade dos eventos? Teriam os métodos mistos uma contribuição à limitações enfrentadas pela interdisciplinaridade?

\section{Métodos mistos para a interdisciplinaridade?}

A expressão métodos mistos, mais comumente pronunciada em inglês - mixed methods - ou ainda Mixed Methods Research, se caracteriza como um modo de pesquisa presente em áreas de saúde, educação, psicologia, ciências sociais e ciências sociais aplicadas em que métodos quantitativos e qualitativos são articulados, combinados. O termo bastante empregado em publicações internacionais, mas não no Brasil, onde existe a preferência para um uso não institucionalizado sobre a conjunção "quali-quanti". Para vários autores, como em Tashakhori e Teddlie (2003) e em Johnson, Onwuegbuzie e Turner (2007), os métodos mistos não se apresentam como uma aproximação localizada entre diferentes metodologias, mas como um terceiro paradigma de pesquisa institucionalizado pela articulação entre o "primeiro", o quantitativa e o "segundo", o qualitativo.

Essa busca pelo status de "campo" científico pode ser observado numa quantidade significativa de publicações como períodicos (como, por exemplo, Journal of Mixed Methods Research Field Methods, Quality and Quantity, International Journal of Multiple Research Approaches, dentre outros) e manuais (Tashakkori e Teddlie, 2003). Uma pesquisa ampla com o termo "mixed methods" no sítio ISI-WebofScience, com busca por título e tema, capturou, em junho de 2013, 4029 artigos, tendo suas primeiras publicações no ano de 1978 (Belytschko et al.,1978; Canuto, 1978). No interior das ciências sociais foram publicados, no mesmo sítio, 460 artigos, com o estudo pioneiro publicado em 1993 (Russek e Weinberg, 1993). 
Essa quantidade significativa de trabalhos acadêmicos demonstra a capacidade de se abordar, não apenas a aplicação da estratégia combinada de métodos aos mais variados objetos científicos, como também, a reflexão sistemática sobre as formas de aplicação de métodos combinados, assim como as implicações de seus resultados no confronto com perspectivas unidimensionais.

Dessa diversidade, Greene, Caracelli e Graham (1989), identificam cinco de tipos fundamentais de aplicação de métodos combinados: triangulação, onde se busca, através de diferentes estratégias de pesquisa, uma convergência de resultados; complementaridade, procedimento de confirmação dos resultados de um método por outro; desenvolvimento, quando os resultados de uma pesquisa potencializam ou abrem novas frentes de pesquisa; iniciação, quando o uso de diferentes métodos é derivado da exploração de contradições e paradoxos resultantes da aplicação de um dado método; e, expansão, quando métodos mistos são utilizados para se abranger diferentes aspectos de um tema de pesquisa, adquirindo, assim, uma configuração metodológica. A profusão de iniciativas métodos mistos tem levado, em paralelo, à emergência de tentativas de sistematização espistemológica dessas iniciativas, como evidencia Creswell e Plano Clark (2011). Essas tentativas demonstram um anseio de se fomentar a edificação de um campo científico capaz de conduzir, para além de uma prática fortuita em grupos de pesquisa, a resultados de pesquisa cada vez mais significativos. A despeito da forma que assumem, nelas predomina o caráter pragmático como se pode verificar em Onwuegbuzie e Turner (2007) no sentido de que as várias perspectivas de combinação são utilizadas mais em função dos resultados que ela potencialmente oferece do que uma adoção prévia do pesquisador em relação a esta ou aquela forma de articulação metodológica. Assim, a combinação entre métodos de pesquisa quantitativa e de métodos de pesquisa qualitativa é geralmente vista como forma de balancear os pontos fortes de tais métodos, compensando suas fraquezas de tal modo a produzir melhores dados e melhores análises (Axinn \& Pearce, 2006). 
|98|

Os métodos mistos e a interdisciplinaridade nas....

Contudo, a perspectiva pragmática, utilitária, não está isenta às críticas, sobretudo por parte das ciências sociais. Teddlie e Tashakkori (2012) defendem a existência de um conjunto de características centrais ao campo (ecletismo, pluralismo diversidade e continuidade), o que possibilitaria compreender os métodos mistos como terreno de convivência entre paradigmas diferentes. Corrobora com essa perspectiva a defesa de Mertens (2010) à perspectiva da divergência como motivação para articulação entre métodos. Do mesmo modo, pensar o pragmatismo como uma base filosófica comum onde diferentes métodos podem se encontrar, reduz a possibilidade de confronto entre perspectivas filosóficas/ epistemológicas diferenciadas e mesmos contrastantes (Biesta, 2010)

Do ponto de vista das ciências sociais, o uso de métodos combinados parece preceder o seu reconhecimento como forma conceitual (Pearce, 2012). Presente desde os clássicos, como por exemplo, Marx (1990), em sua articulação entre análise de documentos e 'participação' para entendimento da França de 1848, ou Malinowski (1976) em sua combinação de observação direta e coleta de dados estatísticos para análise da sociedade trabriandesa, os métodos mistos parecem coadunar com visão weberiana de que a sociologia - e, por extensão, as ciênciais sociais - se configura pela sobreposição de posturas metodológicas compreensivas e explicativas diante dos fenômenos sociais (Weber, 1996). Esse posicionamento apresenta reflexo na contemporaneidade através, por exemplo, da teoria da estruturação (Giddens, 1984) em sua tentativa de articular "agência" e "estrutura" social. A demografia, por sua vez, principalmente em sua vertente sociodemográfica, tem apresentado uma profícua produção em torno das diferentes possibilidades metodológicas de articulação entre volume e distribuição ou entre uso e ocupação do solo (Hogan, 2007; Liverman, 1998; D'Antona et al. 2008).

Assim, podemos reconhecer, entre os propositores dos métodos mistos como um campo institucionalizado, uma tensão acerca do "chão" epistemológico no qual a abordagem deve ser edificada: o pragmatismo unificador ou pluralismo filosófico? 
Se a primeira prescinde da diversidade epistemológica e teórica, conferindo aos métodos certo descolamento de suas origens conceituais em prol da adequação ao fenômeno a ser apropriado, a segunda compreende métodos mistos como uma grande encruzilha epistêmica, no qual a complexidade deve ser enfrentada da forma mais ampla possível, admitindo-se, assim, uma multiplicidade de perspectivas. Ambas procuram possibilitar aos "mixed methods" o status de "terceiro campo" metodológico. Porém, se a primeira aponta para uma conformação à institucionalidade disciplinar, por não colocar em xeque a constituição disciplinar de onde se originam pesquisas combinadas, a segunda abre-se a tentativa colocar diferentes culturas epistêmicas (em suas práticas, orientações conceituais e concepções teóricas) (Knorr-Cetina, 1999) em diálogo.

\section{Considerações finais}

A partir das questões levantadas nos dois exercícios aqui apresentados, como podemos responder as questões que animaram esse artigo? Vimos, por um lado, que disciplinaridade e interdisciplinaridade constituem tanto a tensão produtora do projeto racionalizador moderno quanto às consequências desse projeto na contemporaneidade. Classificamos, na forma de um recurso heurístico provisório, essa tensão por duas perspectivas distintas: interdisciplinaridade formativa e interdisciplinaridade reflexiva. Do mesmo modo, desenvolvemos uma reflexão acerca dos limites da interdisciplinaridade reflexiva, ainda atada, talvez, pela lógica da interdisciplinaridade formativa: um diálogo que ocorre apenas entre os que comungam do mesmo registro epistemológico, gerando assim, uma continuidade colaborativa ou uma "interdisciplinaridade disciplinar". Esta última, nas situações anteriormente analisadas, limitou iniciativas de interdisciplinaridade em zonas e áreas de atuação cinzentas, instaladas nos interstícios de fenômenos híbridos. 
| $100 \mid$

Os métodos mistos e a interdisciplinaridade nas....

Por outro lado, observou-se que o debate epistemológico sobre métodos combinados se configura com tentativa de constituição de um corpus conceitual que possibilite a definição de uma terceira dimensão metodológica, para além da quantitativa e da qualitativa. Esse ponto pacífico entre a maioria dos estudiosos do tema está na superfície de um debate subjacente, de fundo, que se refere à base filosófica. Nesse sentido, há um debate entre uma perspectiva pragmática - na qual os estilos de aplicação devem ser ordenados segundo os interesses imediatos de enfrentamento científico aos fenômenos que se pretende apreender-e a perspectiva do pluralismo paradigmático e epistemológico, no qual se advoga no somente a "convergência", mas também, a "divergência" entre construções teórico-metodológicas diferenciadas que possam acompanhar os processos de combinação metodológica. Essa última perspectiva abre a possibilidade dos processos de aplicação se constituir em fio condutor para o contraste perspectivado em diferentes perspectivas epistêmicas.

Se, por um lado, configura-se uma "interdisciplinaridade disciplinar" e, por outro, uma combinação metodológica pluralista orientada para os interstícios, o estreitamento entre as perspectivas podem contribuir de forma interessante para uma interdisciplinaridade de "confronto e enfrentamento" entre ciências que se mantêm tradicionalmente distantes. Ou seja, um possível caminho para o estabelecimento de uma interdisciplinaridade reflexiva, adequada à apreensão da complexidade, pode se efetivar a partir da busca entre a convivência de diferentes métodos e seus correspondentes arcabouços teórico-metodológicos, a partir de uma postura de aprendizado mútuo entre diferentes ciências naqueles aspectos onde uma dada disciplinaridade não apreende. Para além de uma interdisciplinaridade que busca afinidades eletivas (Weber, 1996) que, no limite, converge para o aparecimento de novas disciplinaridades, os métodos mistos podem contribuir para uma interdisciplinaridade orientada paras as "discrepâncias eletivas", onde o ponto de partida para o "diálogo" é o estranhamento. 


\section{Referências}

AXINN, W. G.; PEARCE, L. D. Mixed method data collection strategies. New York: Cambridge University Press. 2006.

BATESON, G. Steps to Ecology of Mind: Collected Essays in: Anthropology, Psychiatry, Evolution, and Epistemology. Nova Iorque: Ballantine Books. 1972.

BECK, U. Sociedade de Risco: rumo a uma outra modernidade. São Paulo: Editora 34, 2010.

BELYTSCHKO, T.; YEN, H. J.; MULLEN, R. Mixed methods for time integration. Computer Methods in Applied Mechanics and Engineering, v. 17-8, n. FEB, p. 259-275, 1978.

BIESTA, G. J. J. Pragmatism and the philosophical foundations of mixed methods research. In A. TASHAKKORI \& C. TEDDLIE (eds), Handbook of mixed methods in social and behavioral research. Thousand Oaks, CA: Sage. 2010.

CANUTO, C. Eigenvalue approximations by mixed methods. Rairo-Analyse Numerique-Numerical Analysis, v. 12, n. 1, p. 2750, 1978.

D'ANTONA, A. O. et al. Collecting Sketch Maps to understand property land use and land cover in large surveys. Field Methods, v. 20, p. 66-84, 2008.

DESCOLA, P.; PÁLSSON, G. "Introducción", In: DESCOLA, P. e PÁLSSON, G. (Coord). Naturaleza e Sociedad. Perspectivas Antropológicas. México: Siglo Veintiuno Editores. 2001.

DUPUY, J.-P. Na origem das ciências cognitivas. São Paulo: Editora da Universidade Estadual Paulista, 1996.

DURKHEIM, E. Regras do Método Sociológico. São Paulo: Companhia Editora Nacional. 1972.

GIDDENS, Anthony. A constituição da sociedade. São Paulo. Martins Fontes, 1984. 
$\mid 102$ |

Os métodos mistos e a interdisciplinaridade nas....

GREENE, J. C.; CARACELLI, V. J.; GRAHAM, W. F. Toward a Conceptual Framework for Mixed-method Evaluation Designs. In: Educational Evaluation and Policy Analysis, Vol 11, No. 3, pp 255-274. 1989.

HEIMS, S. J. The Cybernetics Group. Cambridge, Mass.: MIT Press, 1991.

HOGAN D. J. (Org.). Dinâmica populacional e mudança ambiental: cenários para o desenvolvimento brasileiro. Campinas: Nepo/ Unfpa, 2007.

HOWE, R. Max Weber's elective affinities: Sociology within the bounds of pure reason. American Journal of Sociology, n. 84, 1978.

INGOLD, T. Being alive: essays on movement, knowledge and description. Oxford: Routledge, 2011.

JOHNSON, B.; ONWUEGBUZIE, A.; TURNER, L. Toward a definition of mixed methods research. Journal of Mixed Methods Research. 1 (2), 112-133. 2007.

JOHNSON, R. B.; ONWUEGBUZIE, A. J. Mixed methods research: A research paradigm whose time has come. In: Educational Researcher, 33(7), 14-26. 2004.

KLEIN, J. T. Interdisciplinarity: History, Theory, and Practice. Detroit: Wayne State University Press, 1990.

KNORR-CETINA, K. Epistemic Cultures: How the Sciences Make Knowledge. Cambridge, MA: Harvard University Press, 1999.

LATOUR, B. Jamais Fomos Modernos: Ensaio de Antropologia Simétrica. Tradução de Carlos Irineu da Costa. RJ: Editora 34. 1994.

LÉVI-STRAUSS, C. Antropologia estrutural. Tempo Brasileiro, RJ, 1985.

LIVERMAN, D. et al. People and Pixels: Linking Remote Sensing and Social Science. Washington, D. C.: National Academy Press, 1998. 
MALINOWSKI, B. Argonautas do pacifico ocidental: um relato do empreendimento e da aventura dos nativos nos arquipélagos da Nova Guiné melanésia. São Paulo: Abril Cultural, 1976.

MARX, K. O Dezoito Brumário de Luis Bonaparte. São Paulo. Edições Mandacaru. 1990.

MORAN, E.F. Adaptabilidade humana: uma introdução à antropologia ecológica. São Paulo: EDUSP, 1994.

MORIN, E. O Paradigma Perdido: a natureza humana. Portugal, Publicações Europa-América, 1973.

PEARCE, L. D. Mixed Methods Inquiry in Sociology. In: American Behavioral Scientist American Behavioral Scientist, 56(6), 829848. 2012.

PLANO CLARK, V. L. Designing and conducting mixed methods research (2nd ed.). Thousand Oaks, CA: Sage. 2011.

SILVA JÚNIOR, R. D. ; DANTONA, A. O. As propostas de sustentabilidade em demografia uma análise sobre a relação entre especialização científica e fenômenos híbridos. In: ENCONTRO NACIONAL DE ESTUDOS POPULACIONAIS, 28, 2012, Águas de Lindoia. Anais do XVIII Encontro Nacional de Estudos Populacionais. Águas de Lindóia: ABEP. 2012.

SILVA JÚNIOR, R. D. Sustentabilidade, um tema híbrido sob olhares purificados uma análise sociológica sobre a produção científica na ecologia e sociologia. In: ENCONTRO ANUAL DA ASSOCIAÇÃO NACIONAL DE PÓS-GRADUAÇÃO E PESQUISA EM CIÊNCIAS SOCIAIS, 36., 2012, Águas de Lindoia. Anais do $36^{\circ}$. Encontro Anual da Associação Nacional de Pós-Graduação e Pesquisa em Ciências Sociais. Águas de Lindóia: ANPOCS, 2012.

SILVA JUNIOR, R.; FERREIRA, L. C. Sustentabilidade na era das conferências sobre meio ambiente e desenvolvimento - um olhar para ecologia e economia. Ambiente e Sociedade. São Paulo, v. 16, n. 1, mar. 2013. 
TASHAKKORI, A.TEDDLIE, C. (Eds.), Handbook of mixed methods in social \& behavioral research. Thousand Oaks, CA: Sage. 2003

TEDDLIE C.; TASHAKKORI, A. Common "Core" Characteristics of Mixed Methods Research. American Behavioral Scientist. 56(6) $774-788.2012$.

\section{Bibliografia Pesquisada:}

BAZELEY, P. Integrating Data Analyses in Mixed Methods Research. Journal of Mixed Methods Research, v. 3, n. 3, p. 203207, Jul 2009. ISSN 1558-6898. Disponível em: <<Go to ISI>:// WOS:000267197100001 >.

BELL, R. A. et al. Encouraging patients with depressive symptoms to seek care: A mixed methods approach to message development. Patient Education and Counseling, v. 78, n. 2, p. 198-205, Feb 2010. ISSN 0738-3991. Disponível em: $<<$ Go to ISI $>$ ://WOS:000275010300012 >.

BERNARDI, L.; KEIM, S.; VON DER LIPPE, H. Social Influences on Fertility A Comparative Mixed Methods Study in Eastern and Western Germany. Journal of Mixed Methods Research, v. 1, n. 1, p. 23-47, Jan 2007. ISSN 1558-6898. Disponível em: <<Go to ISI $>$ ://WOS:000208042100003 >.

BROTTO, L. A. et al. Asexuality: A Mixed-Methods Approach. Archives of Sexual Behavior, v. 39, n. 3, p. 599-618, Jun 2010. ISSN 0004-0002. Disponível em: < <Go to ISI>:// WOS:000276739400006 >.

CASTRO, F. G. et al. A Methodology for Conducting Integrative Mixed Methods Research and Data Analyses. Journal of Mixed Methods Research, v. 4, n. 4, p. 342-360, Oct 2010. ISSN 15586898. Disponível em: <<Go to ISI $>$ ://WOS:000282037000005 >.

COLLINS, K. M. T.; ONWUEGBUZIE, A. J.; JIAO, Q. G. A Mixed Methods Investigation of Mixed Methods Sampling Designs 
in Social and Health Science Research. Journal of Mixed Methods Research, v. 1, n. 3, p. 267-294, Jul 2007. ISSN 15586898. Disponível em: <<Go to ISI>://WOS:000208042300005>.

CRESWELL, J. W. Mapping the Field of Mixed Methods Research. Journal of Mixed Methods Research, v. 3, n. 2, p. 95-108, Apr 2009. ISSN 1558-6898. Disponível em: <<Go to ISI $>$ :// WOS:000264377500001 >.

CRESWELL, J. W.; TASHAKKORI, A. Developing Publishable Mixed Methods Manuscripts. Journal of Mixed Methods Research, v. 1, n. 2, p. 107-111, Apr 2007a. ISSN 1558-6898. Disponível em: <<Go to ISI $>$ ://WOS:000208042200001 >.

CRESWELL, J. W.; TASHAKKORI, A. Differing Perspectives on Mixed Methods Research. Journal of Mixed Methods Research, v. 1, n. 4, p. 303-308, Oct 2007b. ISSN 1558-6898. Disponível em: <<Go to ISI $>$ ://WOS:000208042400001 >.

DELLINGER, A. B.; LEECH, N. L. Toward a Unified Validation Framework in Mixed Methods Research. Journal of Mixed Methods Research, v. 1, n. 4, p. 309-332, Oct 2007. ISSN 15586898. Disponível em: <<Go to ISI>://WOS:000208042400002 >.

DENSCOMBE, M. Communities of practice - A research paradigm for the mixed methods approach. Journal of Mixed Methods Research, v. 2, n. 3, p. 270-283, Jul 2008. ISSN 1558-6898. Disponível em: <<Go to ISI $>$ ://WOS:000259070200005 >.

DENZIN, N. K. Moments, Mixed Methods, and Paradigm Dialogs. Qualitative Inquiry, v. 16, n. 6, p. 419-427, Jul 2010. ISSN 10778004. Disponível em: <<Go to ISI>://WOS:000278278100002 >.

FEILZER, M. Y. Doing Mixed Methods Research Pragmatically: Implications for the Rediscovery of Pragmatism as a Research Paradigm. Journal of Mixed Methods Research, v. 4, n. 1, p. 6-16, Jan 2010. ISSN 1558-6898. Disponível em: < <Go to ISI>:// WOS:000273149100002 >. 
GREENE, J. C. Is mixed methods social inquiry a distinctive methodology? Journal of Mixed Methods Research, v. 2, n. 1, p. 7-22, Jan 2008. ISSN 1558-6898. Disponível em: <<Go to ISI >:// WOS:000259069600002 >.

IVANKOVA, N. V.; CRESWELL, J. W.; STICK, S. L. Using mixedmethods sequential explanatory design: From theory to practice. Field Methods, v. 18, n. 1, p. 3-20, Feb 2006. ISSN 1525822X. Disponível em: <<Go to ISI>://WOS:000242853100001 >.

JOHNSON, R. B.; ONWUEGBUZIE, A. J.; TURNER, L. A. Toward a Definition of Mixed Methods Research. Journal of Mixed Methods Research, v. 1, n. 2, p. 112-133, Apr 2007. ISSN 15586898. Disponível em: <<Go to ISI >://WOS:000208042200002 >.

LEECH, N. L.; ONWUEGBUZIE, A. J. A typology of mixed methods research designs. Quality $\mathcal{E}$ Quantity, v. 43, n. 2, p. 265-275, Mar 2009. ISSN 0033-5177. Disponível em: $<<$ Go to ISI $>: / /$ WOS:000262652900008 >.

LI, X. et al. The Effect of Online Information Search on Image Development Insights from a Mixed-Methods Study. Journal of Travel Research, v. 48, n. 1, p. 45-57, Aug 2009. ISSN 00472875. Disponível em: <<Go to ISI>://WOS:000268025900004 >.

MERTENS, D. M. Transformative Paradigm Mixed Methods and Social Justice. Journal of Mixed Methods Research, v. 1, n. 3, p. 212-225, Jul 2007. ISSN 1558-6898. Disponível em: <<Go to ISI $>: / / W O S: 000208042300002>$.

MISTRY, R. S. et al. Expanding the family economic stress model: Insights from a mixed-methods approach. Journal of Marriage and Family, v. 70, n. 1, p. 196-209, Feb 2008. ISSN 0022-2445. Disponível em: < <Go to ISI >://WOS:000253314900015 >.

MUSTANSKI, B.; LYONS, T.; GARCIA, S. C. Internet Use and Sexual Health of Young Men Who Have Sex with Men: A Mixed-Methods Study. Archives of Sexual Behavior, v. 40, n. 2, p. 289-300, Apr 2011. ISSN 0004-0002. Disponível em: $<<$ Go to ISI $>$ //WOS:000289795500012 >. 
NASTASI, B. K. et al. Mixed Methods in Intervention Research Theory to Adaptation. Journal of Mixed Methods Research, v. 1, n. 2, p. 164-182, Apr 2007. ISSN 1558-6898. Disponível em: < $<$ Go to ISI $>$ ://WOS:000208042200005 >.

O'CATHAIN, A. Mixed Methods Research in the Health Sciences A Quiet Revolution. Journal of Mixed Methods Research, v. 3, n. 1, p. 3-6, Jan 2009. ISSN 1558-6898. Disponível em: <<Go to ISI $>/ /$ WOS:000261492500001 >.

O'CATHAIN, A.; MURPHY, E.; NICHOLL, J. Integration and Publications as Indicators of "Yield" From Mixed Methods Studies. Journal of Mixed Methods Research, v. 1, n. 2, p. 147163, Apr 2007. ISSN 1558-6898. Disponível em: <<Go to ISI>:// WOS:000208042200004 >.

SALE, J. E. M.; LOHFELD, L. H.; BRAZIL, K. Revisiting the quantitative-qualitative debate: Implications for mixedmethods research. Quality \& Quantity, v. 36, n. 1, p. 43-53, Feb 2002. ISSN 0033-5177. Disponível em: < <Go to ISI >:// WOS:000174570600003 >.

TASHAKKORI, A.; CRESWELL, J. W. Exploring the Nature of Research Questions in Mixed Methods Research. Journal of Mixed Methods Research, v. 1, n. 3, p. 207-211, Jul 2007a. ISSN 1558-6898. Disponível em: < <Go to ISI>:// WOS:000208042300001 >.

TASHAKKORI, A.; CRESWELL, J. W. The New Era of Mixed Methods. Journal of Mixed Methods Research, v. 1, n. 1, p. 3-7, Jan 2007b. ISSN 1558-6898. Disponível em: <<Go to ISI >:// WOS:000208042100001 >.

TEDDLIE, C.; YU, F. Mixed Methods Sampling A Typology With Examples. Journal of Mixed Methods Research, v. 1, n. 1, p. 77100, Jan 2007. ISSN 1558-6898. Disponível em: < <Go to ISI $>$ :// WOS:000208042100005 >.

TROCHIM, W. M. et al. The evaluation of large research initiatives - A participatory integrative mixed-methods 
$\mid 108$ |

Os métodos mistos e a interdisciplinaridade nas....

approach. American Journal of Evaluation, v. 29, n. 1, p. 8-28, Mar 2008. ISSN 1098-2140. Disponível em: < <Go to ISI $>$ :// WOS:000253203200001 >.

WOOLLEY, C. M. Meeting the Mixed Methods Challenge of Integration in a Sociological Study of Structure and Agency. Journal of Mixed Methods Research, v. 3, n. 1, p. 7-25, Jan 2009. ISSN 1558-6898. Disponível em: < <Go to ISI $>/ /$ WOS:000261492500002 >. 